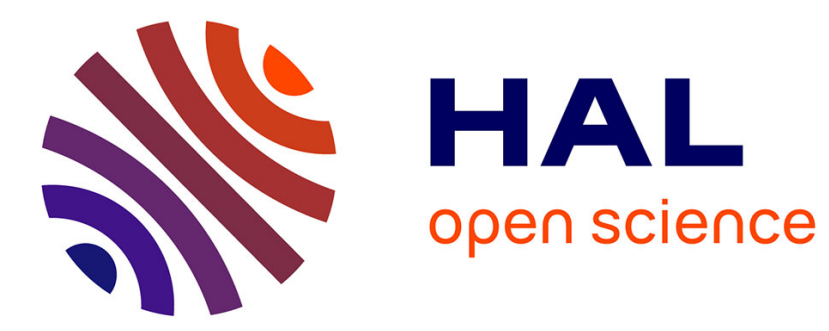

\title{
Statistical Classification for Heterogeneous Polarimetric SAR Images
}

\author{
Pierre Formont, Frédéric Pascal, Gabriel Vasile, Jean-Philippe Ovarlez, \\ Laurent Ferro-Famil
}

\section{To cite this version:}

Pierre Formont, Frédéric Pascal, Gabriel Vasile, Jean-Philippe Ovarlez, Laurent Ferro-Famil. Statistical Classification for Heterogeneous Polarimetric SAR Images. IEEE Journal of Selected Topics in Signal Processing, 2011, 5 (3), pp.567 - 576. 10.1109/JSTSP.2010.2101579 . hal-00638829

\section{HAL Id: hal-00638829 \\ https://hal.science/hal-00638829}

Submitted on 7 Nov 2011

HAL is a multi-disciplinary open access archive for the deposit and dissemination of scientific research documents, whether they are published or not. The documents may come from teaching and research institutions in France or abroad, or from public or private research centers.
L'archive ouverte pluridisciplinaire HAL, est destinée au dépôt et à la diffusion de documents scientifiques de niveau recherche, publiés ou non, émanant des établissements d'enseignement et de recherche français ou étrangers, des laboratoires publics ou privés. 


\title{
Statistical Classification for Heterogeneous Polarimetric SAR Images
}

\author{
Pierre Formont, Student Member, IEEE, Frédéric Pascal, Member, IEEE, Gabriel Vasile, Member, IEEE, \\ Jean-Philippe Ovarlez, Member, IEEE, and Laurent Ferro-Famil, Member, IEEE
}

\begin{abstract}
This paper presents a general approach for highresolution polarimetric SAR data classification in heterogeneous clutter, based on a statistical test of equality of covariance matrices. The Spherically Invariant Random Vector (SIRV) model is used to describe the clutter. Several distance measures, including classical ones used in standard classification methods, can be derived from the general test. The new approach provide a threshold over which pixels are rejected from the image, meaning they are not sufficiently "close" from any existing class. A distance measure using this general approach is derived and tested on a high-resolution polarimetric data set acquired by the ONERA RAMSES system. It is compared to the results of the classical $H-\alpha$ decomposition and Wishart classifier under Gaussian and SIRV assumption. Results show that the new approach rejects all pixels from heterogeneous parts of the scene and classifies its Gaussian parts.
\end{abstract}

Index Terms-Image classification, non-Gaussian modeling, polarimetric synthetic aperture radar, statistical analysis.

\section{INTRODUCTION}

$\mathbf{T}$ HE signal returned by a synthetic aperture radar (SAR) is a complex variable, as it measures both amplitude and phase information of the backscattered signal, producing one complex image for each recording. POLarimetric SAR (POLSAR) systems are able to emit and receive in two (or more) orthogonal polarizations. The interactions between the polarized electromagnetic waves emitted by the radar and the target area can be described thanks to the Sinclair matrix [1]. This matrix can be represented in a vectorized version, the scattering vector. From this vector, its covariance matrix can be computed. Random interferences of the waves scattered within each resolution cell are responsible for the speckle noise [2]. Thus, the backscattered signal from polarimetric SAR data is generally modeled by the multivariate, zero-mean, circular

Manuscript received April 20, 2010; revised September 23, 2010; accepted December 02, 2010. Date of publication December 23, 2010; date of current version May 18, 2011. This work was supported by the DGA. The associate editor coordinating the review of this manuscript and approving it for publication was Prof. Lorenzo Bruzzone.

P. Formont and J.-P. Ovarlez are with ONERA DEMR/TSI, 91761 Palaiseau Cedex, France, and also with Supélec/SONDRA, 91192 Gif-sur-Yvette Cedex, France (e-mail: pierre.formont@ supelec.fr; jean-philippe.ovarlez@onera.fr).

F. Pascal is with Supélec/SONDRA, 91192 Gif-sur-Yvette Cedex, France (e-mail: frederic.pascal@supelec.fr).

G. Vasile is with the GIPSA-lab DIS/SIGMAPHY, Grenoble INP-BP 46, 38402 Saint-Martin-d'Hères, France (e-mail: gabriel.vasile@gipsa-lab.fr; gabriel.vasile@gipsa-lab.grenoble-inp.fr).

L. Ferro-Famil is with the IETR, 35042 Rennes Cedex, France (e-mail: laurent.ferro-famil@univ-rennes1.fr).

Color versions of one or more of the figures in this paper are available online at http://ieeexplore.ieee.org.

Digital Object Identifier 10.1109/JSTSP.2010.2101579 complex Gaussian distribution, which is fully characterized by the covariance matrix.

However, recent POLSAR systems are now capable to acquire very high-resolution images of the Earth's surface, up to decimetric resolution. This increase in resolution leads to a higher scene heterogeneity, particularly in urban areas, where the clutter can no longer be modeled by a Gaussian process [3]. More precisely, the value of each resolution cell corresponds to the sum of all signals reflected by the scatterers within the cell. With higher resolutions, the number of scatterers varies randomly from cell to cell [4]. Thus, the resulting backscattered signal is heterogeneous, as it is locally Gaussian with random power. To take this heterogeneity into account, one can use the Spherically Invariant Random Vector product model, first introduced by Yao [5] for the information theory. The clutter is modeled as compound-Gaussian process, i.e., the product between the square root of a positive random scalar variable, called the texture, and an independent, zero-mean, complex circular Gaussian vector, called the speckle. Since the texture distribution is not specified, this model encompasses an infinity of distributions, notably the Gaussian one and the K-distribution [6]. Furthermore, this model has been validated by several measurements, see, e.g., [6]-[9]. This model has also been recently used by Rangaswamy in [10] to select homogeneous training data for covariance matrix estimation.

Polarimetric SAR images can be used for several applications, mostly for land cover classification. Therefore, polarimetric SAR images classification is an active area of research. Two main approaches appeared in the corresponding literature. The first approach is to classify the images based on their statistical properties: Kong et al. [11] derived a distance measure for single-look complex polarimetric SAR data, which has been extended by Yueh et al. [12] and van Zyl et al. [13] for normalized complex POLSAR data. A distance measure for the multi-look complex case has been proposed by Lee et al. in [14], based on the complex Wishart distribution of the clutter covariance matrix under the Gaussian assumption. The second approach is to classify pixels thanks to their physical characteristics. Several matrix decompositions have been proposed: coherent decompositions, based on the scattering matrix, like the Cameron decomposition [15] or the Krogager decomposition [16], in order to characterize pure scatterers. The distributed scatterers have been studied thanks to incoherent decompositions, i.e., decompositions of the covariance matrix. Freeman et al. [17] proposed such a decomposition. In [18], Cloude et al. proposed the $H-\alpha$ decomposition, based on the eigendecomposition of the covariance matrix. 
These two approaches can be combined. Lee et al. [19] proposed an unsupervised classification algorithm using both physical and statistical properties of the POLSAR image for the classification. More recently, several methods have been considered for the clustering: fuzzy clustering, EM clustering, neural networks but most of these approaches only consider the Gaussian case. Vasile et al. [20] proposed a different approach for the non-Gaussian case, using an adapted model.

The main contribution of this paper is to study the polarimetric SAR classification problem as a statistical test problem on the covariance matrices. First, this approach allows unifying the existing classification methods based on statistical distance measures. Indeed, according to the assumption on the statistical test problem, each distance will be derived again as a test statistic. Second, a more general distance measure will be proposed and will be tested on a polarimetric SAR image. More precisely, the paper is organized as follows: Section II will recap the $H-\alpha$ decomposition and the standard Wishart classifier while Section III presents the heterogeneous model and the improvements on heterogeneous data. Section IV is devoted to the statistical approach based on the theory of statistical tests and validated on real polarimetric SAR image in Section V. Finally, Section VI concludes this paper.

\section{State-Of-The-ARt On Polarimetric Classification IN HoMOgENEOUS ClutTER}

Cloude et al. proposed a decomposition of the covariance matrix in [18], extracting physical parameters from its eigenvalues, named the $H-\alpha$ decomposition. This decomposition will be used as the initialization of the classification algorithms presented in this paper. It is recapped as follows.

\section{A. $H-\alpha$ Decomposition}

Let us denote $\mathbf{k}$ a pixel, i.e., a $m$-dimensional vector containing complex values. In our context, $m$ will be equal to 3 . More precisely, $\mathbf{k}$ can be represented in many bases, the more common ones being the lexicographic basis and the Pauli basis that are defined as follows:

$$
\begin{aligned}
& \mathbf{k}=\left(\begin{array}{c}
H H \\
V V \\
H V
\end{array}\right) \text { in the lexicographic basis } \\
& \mathbf{k}=\frac{1}{\sqrt{2}}\left(\begin{array}{c}
H H+V V \\
H H-V V \\
2 H V
\end{array}\right) \text { in the Pauli basis }
\end{aligned}
$$

where $H H$ (respectively $V V, H V$ ) corresponds to the value acquired with horizontal (respectively vertical, horizontal) polarization in emission and horizontal (respectively vertical, vertical) polarization in reception.

Let us denote $\mathbf{T}$ its covariance matrix, i.e., $\mathbf{T}=E\left[\mathbf{k k}^{H}\right]$, where ${ }^{H}$ is the conjugate transpose operator and $E$ [.] denotes the statistical mean. From the eigendecomposition of the covariance matrix $\mathbf{T}$, two main classification parameters can be extracted: the entropy $H$ and the $\alpha$ angle. The underlying assumption is that there is a dominant scattering mechanism in each cell, which can be characterized by these two parameters.

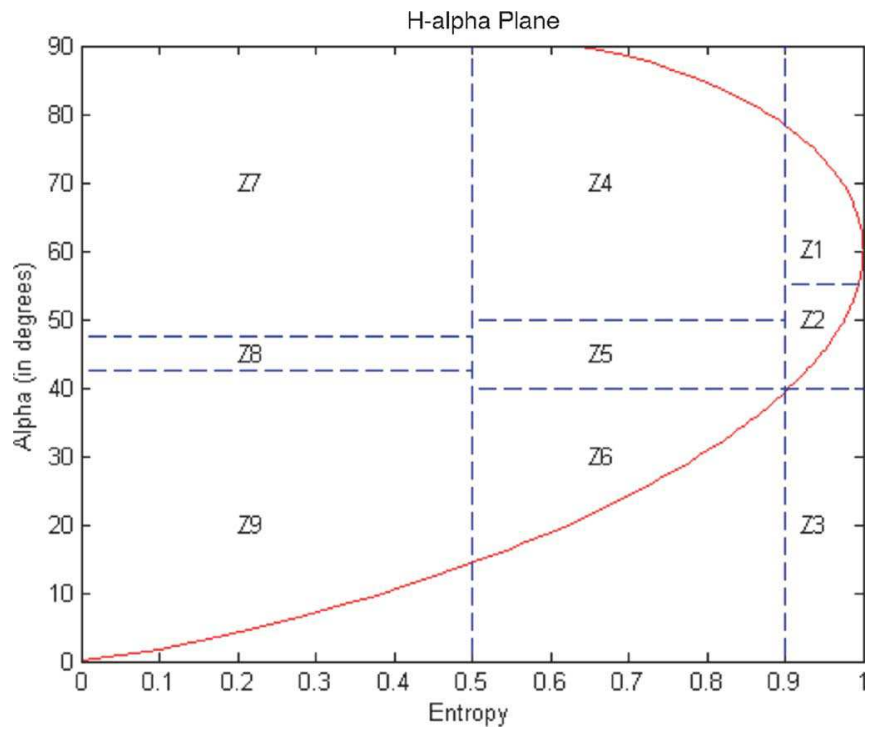

Fig. 1. $H-\alpha$ plane.

$H$ is a function of the eigenvalues $\lambda_{k}$ only, while $\alpha$ is a function of both the eigenvalues and the eigenvectors $\mathbf{u}_{\mathbf{k}}$.

The entropy $H$ measures the chaos inside a resolution cell: if $H$ is close to 0 , there is little chaos, meaning one scattering mechanism is much stronger than all the others in the cell. On the contrary, if $H$ is close to 1 , no mechanism is sufficiently stronger than the others to be dominant inside the cell.

The $\alpha$ angle characterizes the type of the dominant scattering mechanism: for example, when $\alpha$ is close to $0^{\circ}$, the dominant mechanism is a surface diffusion and when $\alpha$ is close to $90^{\circ}$, the dominant mechanism is a dihedral reflection. The relationship between entropy, $\alpha$ angle and scattering mechanisms is represented in Fig. 1.

The $H-\alpha$ plane is separated into eight areas ( $\mathrm{Z} 1$ to $\mathrm{Z9}$, except $\mathrm{Z} 3$ which is not physically feasible), each one corresponding to a specific scattering mechanism. The red line represents the boundary of physically possible $H-\alpha$ couples.

\section{B. Clustering: Wishart Classifier}

One of the main advantages of the $H-\alpha$ decomposition is that it regroups pixels in large clusters. Such information can then be used to classify the image more precisely. Lee et al. [19] proposed an algorithm based on the K-means algorithm, using the $H-\alpha$ decomposition as the algorithm initialization and using the covariance matrix distribution to classify the image. The main assumption is that the class center, which is a covariance matrix, can be computed with higher accuracy, because of the large number of samples contained in each class. Once these class centers are known, each pixel can be reattributed to a class, depending on the distance between its covariance matrix and each class center. The distance used is based on the covariance matrix distribution and is detailed in the following.

1) Wishart Distance: The pixel under consideration, characterized by its target vector $\mathbf{k}$, is supposed to follow a zeromean complex Gaussian distribution denoted $\mathcal{C N}(\mathbf{0}, \mathbf{T})$ with covariance matrix T. Considering an independent and identically distributed (i.i.d.) $N$-sample $\left(\mathbf{k}_{1}, \ldots, \mathbf{k}_{N}\right)$ corresponding 
to a spatial neighborhood ${ }^{1}$ of the pixel $\mathbf{k}$, the maximum-likelihood (ML) estimator of $\mathbf{T}$ is the well-known sample covariance matrix, given by

$$
\widehat{\mathbf{T}}_{\mathrm{SCM}}=\frac{1}{N} \sum_{i=1}^{N} \mathbf{k}_{i} \mathbf{k}_{i}^{H} .
$$

$\widehat{\mathbf{T}}_{\mathrm{SCM}}$ has a complex Wishart distribution [21]:

$p\left(\widehat{\mathbf{T}}_{\mathrm{SCM}}\right)$

$$
=\frac{N^{m(N-m)}\left|\widehat{\mathbf{T}}_{\mathrm{SCM}}\right|^{N-m} \exp \left[-N \operatorname{Tr}\left(\mathbf{T}^{-1} \widehat{\mathbf{T}}_{\mathrm{SCM}}\right)\right]}{K(N, m)|\mathbf{T}|^{N}}
$$

where $K(N, m)=\pi^{m(m-1) / 2} \prod_{i=1}^{m} \Gamma(N-i+1)$ and $\Gamma($.$) is$ the Gamma function [22].

A distance measure, called the Wishart distance, can be derived [14] from this distribution and a Bayes ML procedure

$$
d_{W}\left(\widehat{\mathbf{T}}_{\mathrm{SCM}}, \mathbf{C}_{i}\right)=\ln \frac{\left|\mathbf{C}_{i}\right|}{\left|\widehat{\mathbf{T}}_{\mathrm{SCM}}\right|}+\operatorname{Tr}\left(\mathbf{C}_{i}^{-1} \widehat{\mathbf{T}}_{\mathrm{SCM}}\right)
$$

where $\mathbf{C}_{i}$ is the center of the class $i$.

Algorithm: The subsequent algorithm, based on a classical $\mathrm{K}$-means clustering algorithm is described as follows.

1) Initially classify the image using the $H-\alpha$ decomposition, thus creating eight classes.

2) For $i=1 \ldots 8$, compute the class centers: $\mathbf{C}_{i}=$ $\left(1 / N_{i}\right) \sum_{k=1}^{N_{i}} \widehat{\mathbf{T}}_{\mathrm{SCM}}^{(k)}$, where $N_{i}$ is the number of pixels in the class $i$ and where $\widehat{\mathbf{T}}_{\mathrm{SCM}}^{(k)}$ is the covariance matrix estimator of the $k^{t h}$ pixel in the class $i$.

3) For each pixel, compute the Wishart distances between its covariance matrix and each class center using (2) and assign the pixel to the class whose center minimizes the distance.

4) Check the termination criterion. If it is met, stop the algorithm, else go to step 2.

This approach relies heavily on the Gaussian assumption for pixels distribution and can perform poorly when this assumption is not valid anymore. The case of non Gaussian distributions is addressed in the next section.

\section{State-OF-ThE-ART On Polarimetric Classification IN HETEROGENEOUS ClutTER}

The increase in resolution leads to much smaller resolution cells. This results in a heterogeneous backscattered signal and previous methods may give poor results. It is thus necessary to consider an heterogeneous signal model.

\section{A. SIRV Model}

A SIRV is a compound Gaussian vector, defined as the product of a positive scalar random variable $\tau$ and a $m$-dimensional complex circular Gaussian vector, $\mathbf{x}$. Then the target vector $\mathbf{k}$ can be rewritten as

$$
\mathbf{k}=\sqrt{\tau} \mathbf{x} .
$$

${ }^{1}$ For a rigorous estimation procedure, $N$ independent temporal realizations of the pixel $\mathbf{k}$ should be considered but in practice, those are not available since there is only one image. For that purpose, the spatial independency and the local homogeneity are commonly assumed to have an i.i.d. $N$-sample.
This model exhibits many advantages such as follows.

- $\mathbf{x} \sim \mathcal{C N}(\mathbf{0}, \mathbf{M})$ is called the speckle and represents the polarimetric information contained in the clutter.

- $\tau$ is called the texture and represents the local variations of power from cell to cell, thus the heterogeneity of the clutter.

- As $\tau$ 's probability density function (pdf) is not explicitly specified, a whole range of random processes can be described by this model, including of course the Gaussian one. We can also cite the K-distribution for a Gamma distributed texture, Chi, Rayleigh, Weibull, or Rician pdfs [23].

- Measurements campaigns have been realized to show this model matches well with real data [6], [24].

The SIRV model is uniquely defined up to a multiplicative constant with respect to the covariance matrix $\mathbf{M}$ of the Gaussian kernel. Let $\mathbf{M}_{1}$ and $\mathbf{M}_{2}$ be two covariance matrices such as $\mathbf{M}_{1}=\alpha \mathbf{M}_{2}, \alpha \in \mathbb{R}^{+*}$. The two different sets of parameters $\left\{\mathbf{M}_{1}, \tau\right\}$ and $\left\{\mathbf{M}_{2},(\tau / \sqrt{\alpha})\right\}$ describe the same SIRV. To solve this identification problem, the covariance matrix $\mathbf{M}$ has to be normalized. We choose to normalize it such that $\operatorname{Tr}(\mathbf{M})=m$, where $\operatorname{Tr}($.$) denotes the trace of a matrix.$ This normalization results in transferring all the power information in the texture parameter, while the speckle parameter contains only information about the polarimetric diversity. As a result, the interesting matrix for our study is the normalized covariance matrix of the Gaussian kernel $\mathbf{M}$.

Using this model, Gini et al. [25] and Conte et al. [26] propose the exact ML estimate of $\mathbf{M}$, when assuming that the textures $\tau_{i}$ of each component of the $N$-sample of spatial neighbors are deterministic. It is the solution of the following recursive equation:

$$
\begin{aligned}
\widehat{\mathbf{M}}_{\mathrm{FP}} & =f\left(\widehat{\mathbf{M}}_{\mathrm{FP}}\right)=\frac{m}{N} \sum_{i=1}^{N} \frac{\mathbf{k}_{i} \mathbf{k}_{i}^{H}}{\mathbf{k}_{i}^{H} \widehat{\mathbf{M}}_{\mathrm{FP}}^{-1} \mathbf{k}_{i}} \\
& =\frac{m}{N} \sum_{i=1}^{N} \frac{\mathbf{x}_{i} \mathbf{x}_{i}^{H}}{\mathbf{x}_{i}^{H} \widehat{\mathbf{M}}_{\mathrm{FP}}^{-1} \mathbf{x}_{i}} .
\end{aligned}
$$

The associated recursive algorithm to compute $\widehat{\mathbf{M}}_{\mathrm{FP}}$, the Fixed Point (FP) of $f$ is given by

$$
\widehat{\mathbf{M}}_{\mathrm{FP}}^{(i+1)}=f\left(\widehat{\mathbf{M}}_{\mathrm{FP}}^{(i)}\right) \text {. }
$$
Id.

To initialize this recursive algorithm, one can choose $\mathbf{M}_{\mathrm{FP}}^{(0)}=$

The estimate from (3) holds for stochastic $\tau_{i}$ and becomes an approximate maximum-likelihood (AML) estimate. Pascal et al. [27] have proven the existence and the uniqueness of the fixed point estimator as the solution of (3). They also prove in [27] the convergence of the recursive algorithm, whatever the initialization.

\section{B. SIRV Distance}

This new estimator, adapted to the non-Gaussian case has been used by Vasile et al. [20] to derive a distance measure. The estimation scheme is the same as in the Gaussian case, only the estimator used differs. The resulting distance, called the SIRV distance, is given by the following equation:

$$
d_{S}\left(\widehat{\mathbf{M}}_{\mathrm{FP}}, \mathbf{C}_{i}\right)=\ln \frac{\left|\mathbf{C}_{\mathbf{i}}\right|}{\left|\widehat{\mathbf{M}}_{\mathrm{FP}}\right|}+\frac{m}{N} \sum_{n=1}^{N} \frac{\mathbf{k}_{n}^{H} \mathbf{C}_{i}^{-1} \mathbf{k}_{n}}{\mathbf{k}_{n}^{H} \widehat{\mathbf{M}}_{\mathrm{FP}}^{-1} \mathbf{k}_{n}}
$$

where $\mathbf{C}_{i}$ is the center of the class $i$. 


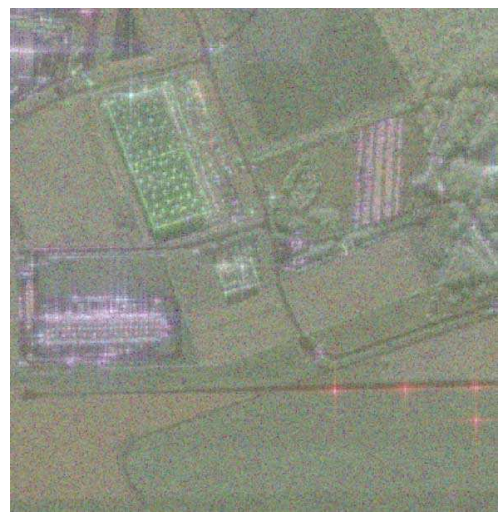

Fig. 2. Amplitude color composition image of the Brétigny region: $k_{1}-k_{3}-k_{2}$.

\section{Results and Discussion}

1) High-Resolution POLSAR Data Set: The data set considered was acquired by the ONERA RAMSES system in Brétigny, France. The acquisition was made in X-band, with a spatial resolution of $1.32 \mathrm{~m}$ in range and $1.38 \mathrm{~m}$ in azimuth. The resulting image is $501 \times 501$ pixels. Fig. 2 shows a color composition of the data in the Pauli basis

$$
\mathbf{k}=\left(\begin{array}{c}
k_{1} \\
k_{2} \\
k_{3}
\end{array}\right)=\frac{1}{\sqrt{2}}\left(\begin{array}{c}
H H+V V \\
H H-V V \\
2 H V
\end{array}\right)
$$

$k_{1}$ is in red, $k_{2}$ in blue, and $k_{3}$ in green.

The polarimetric diversity in this image is high as different features are present: several buildings, a parking lot, different kind of fields, forested areas, roads, etc.

2) $H-\alpha$ Decomposition: Fig. 3 shows the $H-\alpha$ decomposition applied to the data set using the SCM estimate in Fig. 3(a) and the FP estimate in Fig. 3(b). Both results are similar, in terms of visual interpretation, which means that the FP estimate has the same polarimetric properties as the SCM.

3) Classical Wishart Classifier: The Wishart classifier of Section II has been applied on this data set. The results can be seen in Fig. 4.

We can notice that the Gaussian areas are clearly identified: fields in yellow in the bottom part of the image and the blue field in the top part. Urban areas, on the contrary, are a mix on several classes and do not stand out as well. This is due to the fact that the SCM is not a good estimate for heterogeneous areas. Moreover, in [20], it has been shown that the SCM estimate is heavily polluted by the power information.

4) Fixed Point Estimate: Replacing the SCM by the FP estimate in the Wishart Classifier algorithm yields much better results in heterogeneous areas, as can be seen in Fig. 5.

The two big buildings stand out much more in this image than in Fig. 4 and are composed of only one class each. The classification remains satisfactory on most of the Gaussian areas, though it is less good than with the SCM estimate, in terms of separation.

5) SIRV Distance: When replacing the Wishart distance by the SIRV distance in the algorithm, the results, as seen in Fig. 6, stay very close when using the FP estimate. Notice that the classification improvement comes only from the FP estimate and not from the distances.

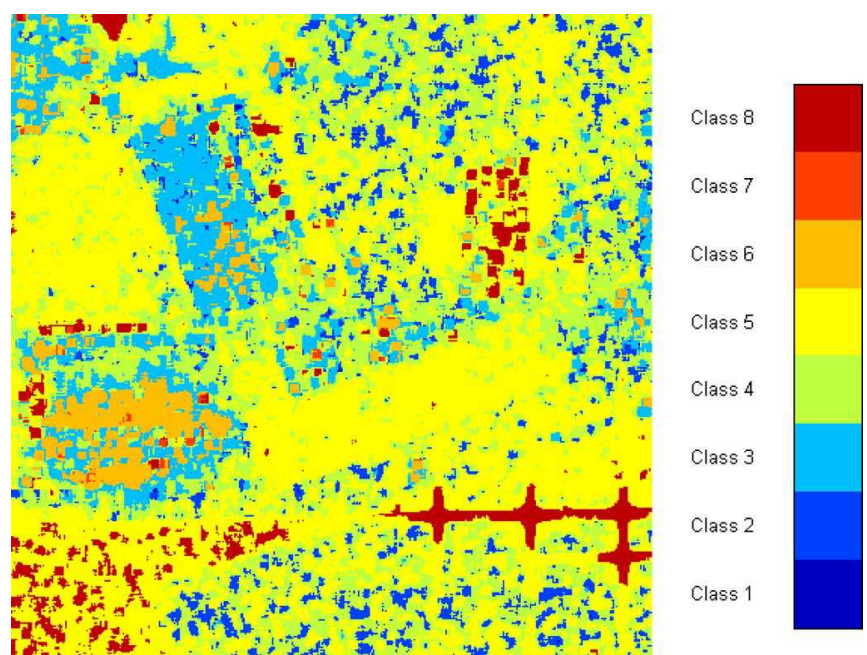

(a)

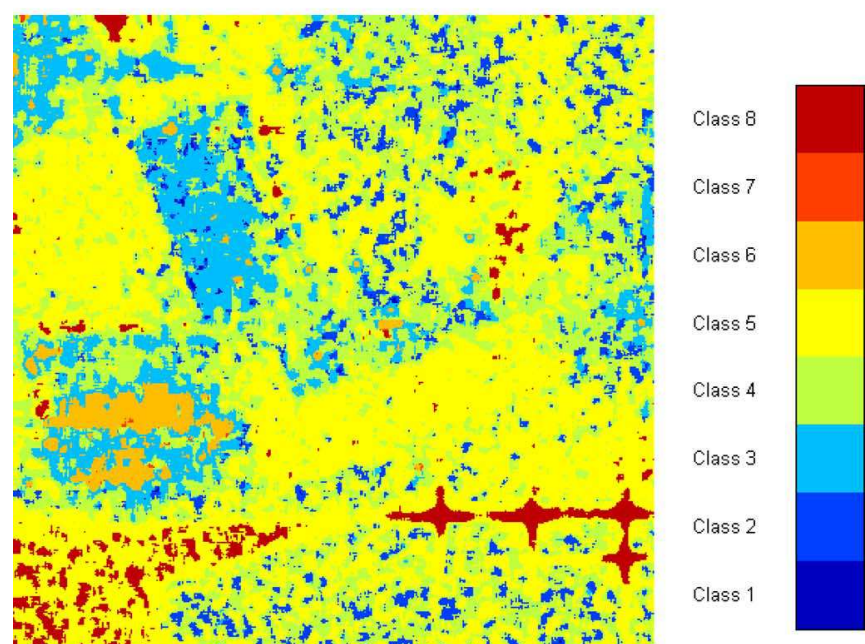

(b)

Fig. 3. $H-\alpha$ decomposition. (a) SCM. (b) FP.

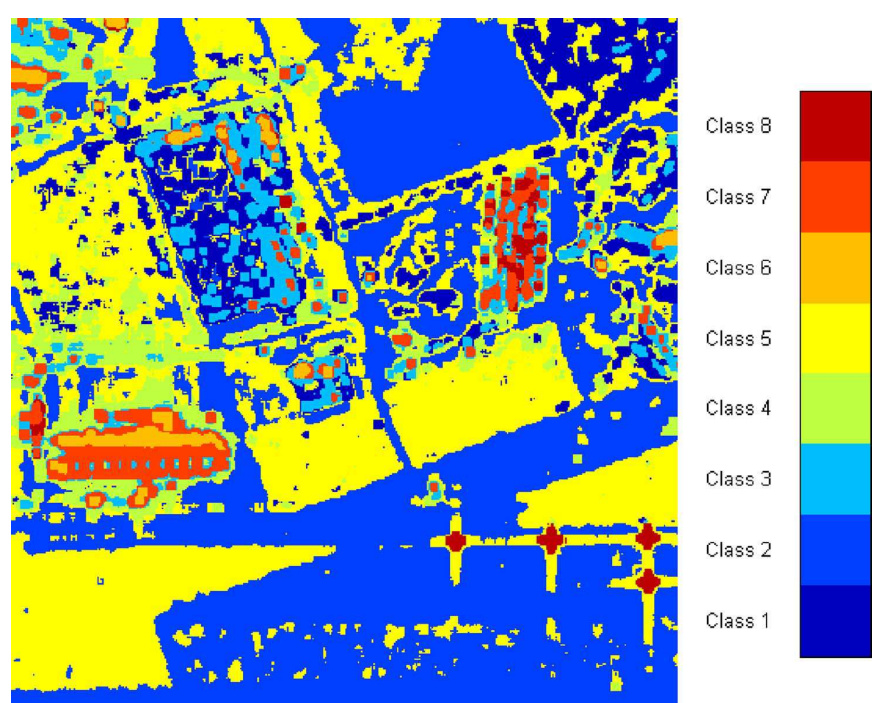

Fig. 4. Wishart classifier, SCM, $H-\alpha$ initialization.

6) $H-\alpha$ Initialization: The use of the $H-\alpha$ decomposition for the initialization may create a bias in the final classification 

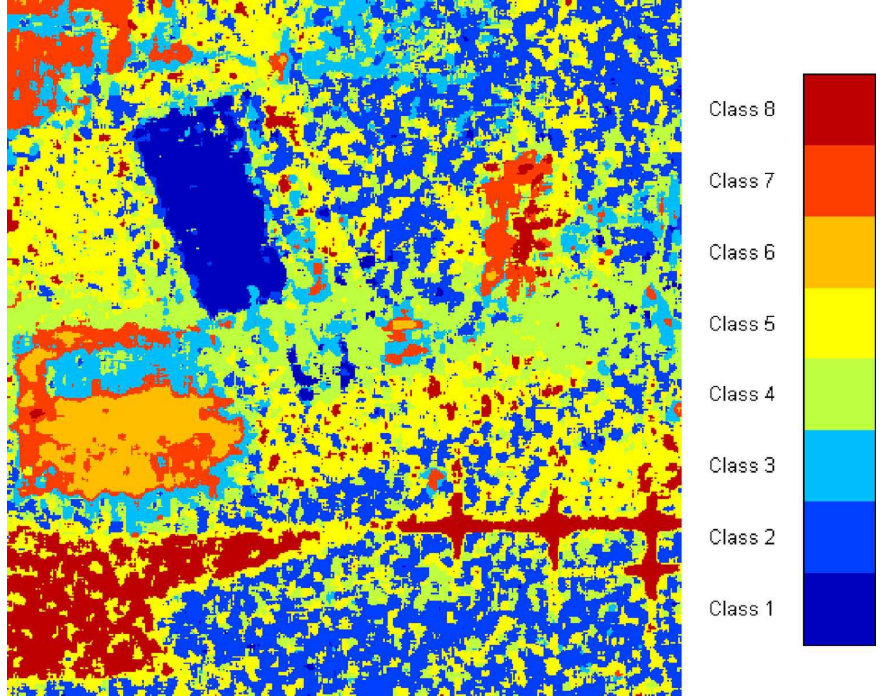

Fig. 5. Wishart classifier, FP, $H-\alpha$ initialization.
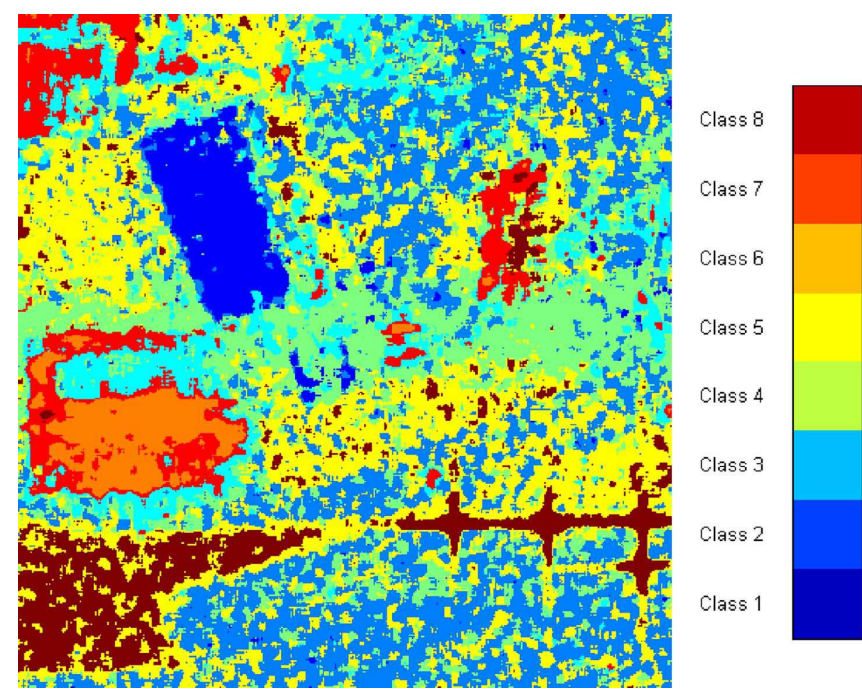

Fig. 6. SIRV classifier, FP, $H-\alpha$ initialization

results, as the pixels are initially clustered into classes thanks to their physical properties, while the distances are a result of the statistical distribution of the clutter. We have investigated the initialization by comparing the end results of the classifier, using $H-\alpha$ initialization or a totally random initialization: each pixel is randomly assigned to 1 out of 8 classes at the start of the classifier. Results can be seen in Fig. 7.

Although the colors do not match, the final classification results are very close to each other. We can therefore dispense with the initial $H-\alpha$ decomposition as it does not improve the end results for this data set.

7) Conclusion: The SCM estimate gives poor results compared to the FP estimate in a non-Gaussian environment. The physical interpretation given by the $H-\alpha$ prior to the classification is not relevant, as a completely random initialization gives the same classification results. On the other hand, the SIRV distance, while optimal for the SIRV case, does not improve the classification in comparison to the Wishart distance and is
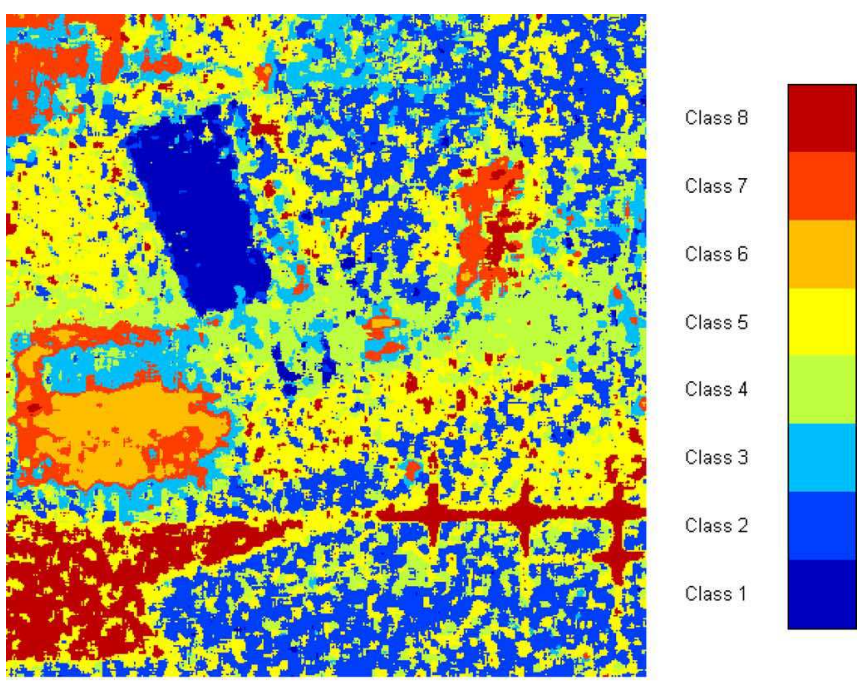

(a)
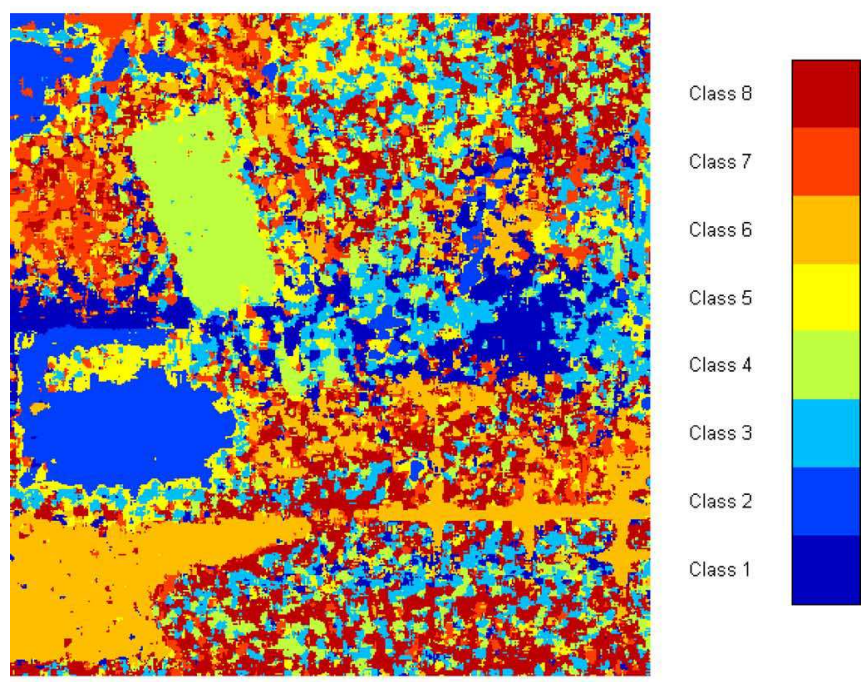

(b)

Fig. 7. Wishart classifier, FP. (a) $H-\alpha$ initialization. (b) Random initialization.

much more computationally heavy. Besides, these methods do not necessarily take into account the possibility of pixels being too far away from every class center. In that case, it makes no sense to add these pixels to any of the classes. In the classical methods, it is possible to take this possibily into account but the threshold has to be set manually. As we want to classify pixels based on their polarimetric properties, i.e., their covariance matrices, we propose to use a statistical test of equality of covariance matrices, which will give a more general approach to the classification problem. This approach will also allow us to rederive the classical Wishart distance and the SIRV distance of [20] more formally according to the hypotheses used in the statistical test problem. These two distances are simply a particular case of the proposed method.

\section{STATisticAl APPROACH FOR POLSAR DATA CLASSIFICATION - MAIN CONTRIBUTION}

This section is devoted to the study of statistical test for equality of covariance matrices and its application to POLSAR 
data classification. A statistical approach to the problem of POLSAR data classification has many advantages.

- It can be applied to both homogeneous and heterogeneous clutter models.

- There is no a priori physical interpretation to the classification process.

- It is a very general approach which particularly contain previous methods.

\section{A. Statistical Framework}

Let $\mathbf{X}_{1}$ and $\mathbf{X}_{2}$ be independent random vectors, such that $\mathbf{X}_{1} \sim \mathcal{L}\left(\mathbf{0}, \mathbf{T}_{1}\right)$ and $\mathbf{X}_{2} \sim \mathcal{L}\left(\mathbf{0}, \mathbf{T}_{2}\right)$, where $\mathcal{L}$ stands for any distribution with the two first moments existing. The goal is to decide if their covariance matrices $\mathbf{T}_{1}$ and $\mathbf{T}_{2}$ are equal. The resulting binary hypothesis test can be written as

$$
\begin{cases}H_{0}: & \mathbf{T}_{1}=\mathbf{T}_{2} \\ H_{1}: & \mathbf{T}_{1} \neq \mathbf{T}_{2}\end{cases}
$$

Let $\mathbf{x}_{1}$ (resp. $\mathbf{x}_{2}$ ) be an observation of $\mathbf{X}_{1}$ (resp. $\mathbf{X}_{2}$ ) and $N=N_{1}+N_{2} \cdot \mathbf{x}_{1}$ and $\mathbf{x}_{2}$ are supposed i.i.d. Let $\mathbf{x}_{1}$ (resp. $\left.\mathbf{x}_{2}\right)$ be of size $N_{1}$ (resp. $N_{2}$ ).

The likelihood function is then

$$
\Lambda=\prod_{i=1}^{2} \operatorname{PDF}\left(\mathbf{x}_{i}\right)
$$

where $\mathbf{x}_{i}^{(k)}$ is the $k^{\text {th }}$ element of the $N_{i}$-sample $\mathbf{x}_{i}$.

This is a more general approach for the pixels classification. Indeed, the idea with previous test is to assign two different pixels in the same class or not.

1) $\mathbf{T}_{2}$ Known: Let us assume that $\mathbf{T}_{2}$ is known and equal to $\mathbf{C}_{i}$, i.e., the center of the class $i$.

- If $\mathbf{X}_{1}$ is Gaussian distributed, the likelihood ratio test statistic derived from (5) easily provides the Wishart distance defined by (2).

- If $\mathbf{X}_{1}$ is SIRV distributed, the likelihood ratio test statistic derived from (5) provides the SIRV distance defined by (4).

In both cases, the approach of statistical tests shows that the corresponding distances used for the polarimetric SAR classification are actually specific test statistics. Thus, to decide that two covariance matrices are equal, it is not sufficient to minimize the distance between these two covariance matrices (done in the previous classification procedure). The minimal distance has to be also smaller than a threshold, directly relied to the distribution of the test statistic and given by the type I error.

2) $\mathbf{T}_{2}$ Unknown: The maximum-likelihood estimator of $\mathbf{T}_{i}$ is

$$
\widehat{\mathbf{T}}_{i}=\frac{1}{N_{i}} \sum_{k=1}^{N_{i}} \mathbf{x}_{i}^{(k)^{H}} \mathbf{x}_{i}^{(k)} .
$$

$\widehat{\mathbf{T}}_{i}$ is the classical SCM defined in (1); hence, it is Wishartdistributed.

Equation (5) needs to be maximized under $H_{0}$, i.e., when $\mathbf{T}_{1}=\mathbf{T}_{2}=\mathbf{T}$. Equation (5) then becomes

$$
\frac{1}{\pi^{m N / 2}|\mathbf{T}|^{N / 2}} \exp \left(-\frac{1}{2} \sum_{i=1}^{2} \sum_{k=1}^{N_{i}} \mathbf{x}_{i}^{(k)}{ }^{H} \mathbf{T}_{i}^{-1} \mathbf{x}_{i}^{(k)}\right) \text {. }
$$

The optimal estimator of $\mathbf{T}$ is

$$
\widehat{\mathbf{T}}=\frac{1}{N}\left(N_{1} \widehat{\mathbf{T}}_{1}+N_{2} \widehat{\mathbf{T}}_{2}\right) .
$$

By replacing (6) in (8) and $\mathbf{T}_{i}$ by its estimate $\widehat{\mathbf{T}}$, the test statistic is

$$
\lambda=\frac{\left|\widehat{\mathbf{T}}_{1}\right|^{N_{1} / 2}\left|\widehat{\mathbf{T}}_{2}\right|^{N_{2} / 2}}{|\widehat{\mathbf{T}}|^{N / 2}} .
$$

Notice the exponents are the size of the samples. Bartlett [28] proposed alternative exponents for the univariate case, replacing the samples size by the degree of freedom of the estimators $\widehat{\mathbf{T}}_{i}$. Equation (9) then becomes

$$
t=\frac{\left|\widehat{\mathbf{T}}_{1}\right|^{\nu_{1} / 2}\left|\widehat{\mathbf{T}}_{2}\right|^{\nu_{2} / 2}}{\left|\widehat{\mathbf{T}}_{t}\right|^{\nu_{t} / 2}}
$$

where $\nu_{i}=N_{i}-1$ are the degrees of freedom of $\widehat{\mathbf{T}}_{i}$ and $\nu_{t}=$ $N-2$, the degree of freedom of $\widehat{\mathbf{T}}$

The statistic $t$ varies between 0 and 1 , with values close to 0 rejecting the null hypothesis, while values close to 1 accept the null hypothesis. To illustrate this, let consider (10) as

$$
t=\left(\frac{\left|\widehat{\mathbf{T}}_{1}\right|}{|\widehat{\mathbf{T}}|}\right)^{\nu_{1} / 2}\left(\frac{\left|\widehat{\mathbf{T}}_{2}\right|}{|\widehat{\mathbf{T}}|}\right)^{\nu_{2} / 2} \text {. }
$$

When $\widehat{\mathbf{T}}_{1}=\widehat{\mathbf{T}}_{2}=\widehat{\mathbf{T}}, t=1$. As the difference between $\widehat{\mathbf{T}}_{1}$ and $\widehat{\mathbf{T}}_{2}$ increases, the smaller factor reduces the product more than the higher factor increases it, thus bringing $t$ closer to 0 .

Box [29], [30] proposed $\chi^{2}$ - and $F$ - approximations for the distribution of $t$. For the $\chi^{2}$-approximation, the statistic he proposed is

$$
\begin{aligned}
u & =-2\left(1-c_{1}\right) \ln (t) \sim \chi^{2}\left(\frac{1}{2}(k-1) m(m+1)\right) \\
\text { where } c_{1} & =\left(\sum_{i=1}^{k} \frac{1}{\nu_{i}}-\frac{1}{\sum_{i=1}^{k} \nu_{i}}\right)\left(\frac{2 m^{2}+3 m-1}{6(k-1)(m+1)}\right)
\end{aligned}
$$

and $\chi^{2}(a)$ denotes the $\chi^{2}$ distribution with $a$ degrees of freedom.

The critical region of the test is

$$
\left\{u>\chi^{2}\left(\frac{1}{2}(k-1) m(m+1), P_{F A}\right)\right\}
$$

where $P_{F A}$ is the type I error, or false-alarm rate. In our case, we set the type I error (accepting the null hypothesis when it is not true, i.e., rejecting the equality of the matrices of the two populations when they are actually equal) to be very low.

3) SIRV Case: The same procedure can be applied in the SIRV case, considering that it was demonstrated by Pascal et $a l$. in [31] that the FP estimate asymptotically has a Wishart distribution behavior with $(m /(m+1)) N$ degrees of freedom. This is a very important property of the FP estimate since all results obtained with the SCM remain valid for the FP estimate due to an asymptotical justification. The SCM estimators $\widehat{\mathbf{T}}_{1}$ and $\widehat{\mathbf{T}}_{2}$ can be replaced by the FP estimates $\widehat{\mathbf{M}}_{1}$ and $\widehat{\mathbf{M}}_{2}$ of the same N-samples $\mathbf{x}_{1}$ and $\mathbf{x}_{2}$ with the correct degrees of freedom. 


\section{Algorithm}

The algorithm used is very similar to the previous algorithm of Section II, except for the fact that the number of classes is not specified at the beginning. We start the algorithm with only one class and increase the number of classes at each iteration. To decide if a pixel is joining a class, the test statistic of (10) is used. The N-samples required to compute the estimation of the covariance matrice are simple boxcar neighorhoods, centered on the pixel under consideration. The classification map is initialized with the $H-\alpha$ decomposition: indeed, we need an initial class that has some sense. We tried initializing the algorithm with one pixel randomly chosen across the image but the end results were not satisfying. Indeed, selecting only one pixel for the initialization leads to very different class centers from one execution of the algorithm to the other. A large number of pixels has to be selected to provide a robust initialization. A quick way to do this is to actually use the $H-\alpha$ decomposition. Note that a random initialization with a large number of pixels randomly chosen yields the same results, as discussed in Section V. One of the largest zones of the $H-\alpha$ decomposition is then chosen as the first class of the algorithm. Its center is computed as

$$
\mathbf{C}_{1}=\frac{1}{N_{1}} \sum_{k=1}^{N_{1}} \widehat{\mathbf{T}}_{\mathrm{SCM}}^{(k)} .
$$

Notice that, for the SIRV case, $\widehat{\mathbf{T}}_{\mathrm{SCM}}$ is replaced by $\widehat{\mathbf{M}}_{\mathrm{FP}}$. All pixels are then reclassified according to the test of (10), used as a distance measure. Equation (11) gives the critical region of the test. In our case, the number of populations, $k$, is 2 and the number of variables, $m$, is 3 so the threshold over which the hypothesis is rejected (i.e., the covariance matrix of the considered pixel is not equal to the covariance matrix of the class) is given by the threshold of a $\chi^{2}$-distribution with 6 degrees of freedom and a type I error the user can define (we chose $10^{-3}$ ). This means that pixels are not automatically put in one of the existing classes. A pixel is assigned to the class who minimizes the distance only if this minimal distance is below the threshold. If the minimal distance is over the threshold, the pixel is put in a rejection class, meaning that it is not close to any of the current classes. The number of classes has then to be incremented and the new class is initialized as the previous rejection class.

The selection of training data for the covariance matrix estimation is made with a $5 \times 5$ boxcar filter around the pixel under consideration. The Estimated Number of Looks $N_{e}^{S}$ in SIRV environment is linked to the Estimated Number of Looks $N_{e}^{G}$ in homogeneous Gaussian environment according to the asymptotic statistical behavior of the normalized Fixed Point Estimate [31]: $N_{e}^{S}=(m /(m+1)) N_{e}^{G}$. For the $5 \times 5$ boxcar, we get $3 / 4 \times 25=18.75$ looks.

The classification procedure is summarized as follows.

1) Initially classify the image using the $H-\alpha$ decomposition. Pick one of the largest zones as the starting class of the algorithm.

2) Compute the class centers $C_{i}$.

3) For each pixel in the image, compute its distance to all the class centers using (10). If the minimal distance is below the threshold, assign the pixel to the corresponding class. Else, put it into the rejection class.

4) Once all pixels have been classified, check the termination criterion. If it is not met, increase the number of classes
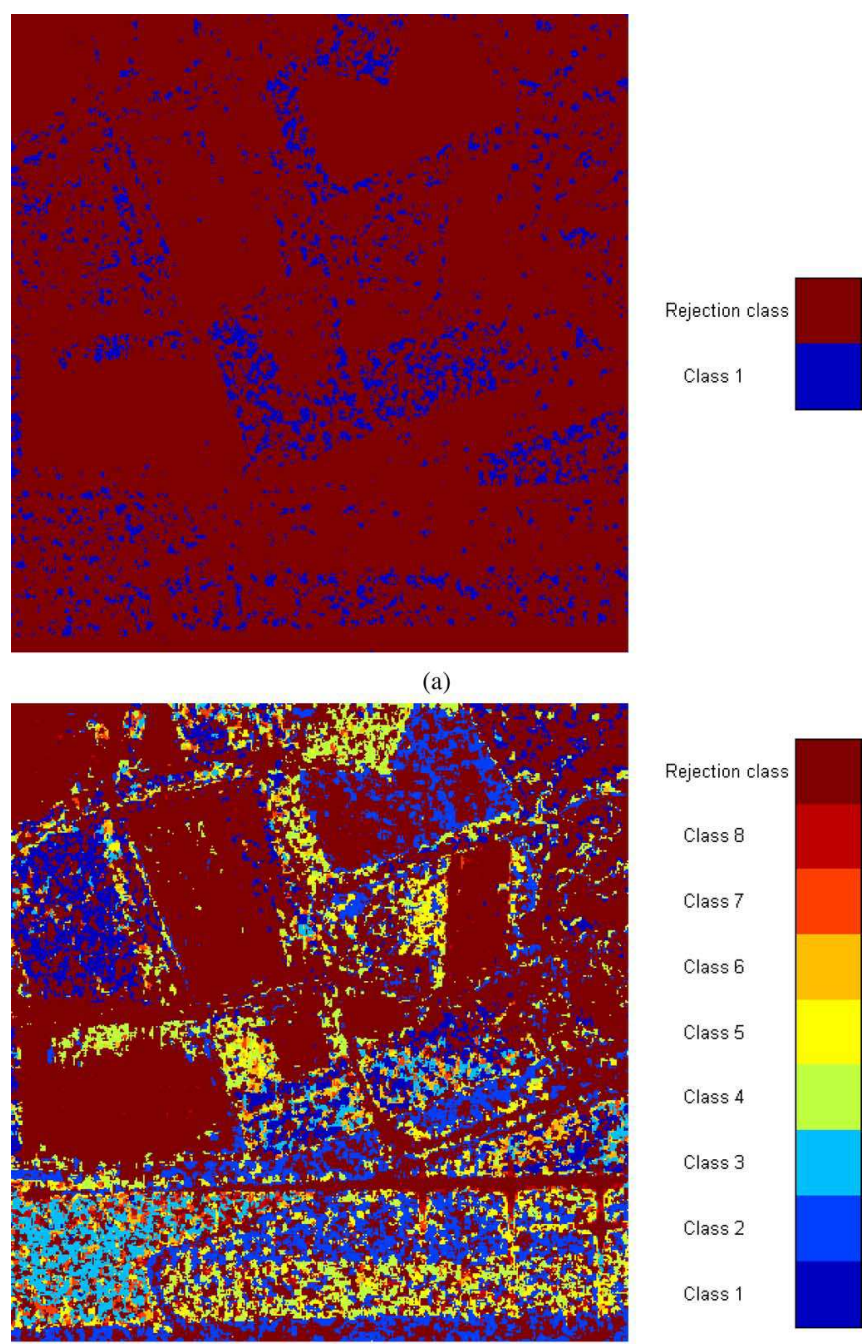

(b)

Fig. 8. Box classifier with SCM and associated colormaps. (a) One class + rejection class. (b) Eight classes + rejection class.

by 1 and go to step 2 . The new class $c_{i+1}$ is defined as the rejection class computed in iteration $(i)$.

Notice that the introduction of a rejection class is more rigorous than the existing methods with respect to the statistical test procedure. Indeed, previous methods assigned a pixel to the class whose center minimizes the distance measure, even if this distance was very high.

\section{RESULTS AND DISCUSSION}

The test statistic of (10) has been applied successfully by Conradsen et al. in [32] for the Gaussian case in change detection. They were able to detect changes more precisely using this fully polarimetric test statistic.

The algorithm proposed in the previous section has been applied on the data described in Section III for classification. The termination criterion chosen is to stop the algorithm at eight classes (plus one rejection class) in order to compare the classification against a Wishart classifier with the standard eight classes from the $H-\alpha$ decomposition.

1) SCM Estimate: Fig. 8 shows the results of the algorithm of Section IV with the SCM estimate of the covariance matrix after 1 iteration on Fig. 8(a) and after eight iterations on Fig. 8(b). 
TABLE I

Number of PiXels in EACH Class at EACH ITERATION - SCM

\begin{tabular}{|c|c|c|c|c|c|c|c|c|c|}
\hline Iteration & Class 1 & Class 2 & Class 3 & Class 4 & Class 5 & Class 6 & Class 7 & Class 8 & Rejection class \\
\hline 1 & 23460 & 0 & 0 & 0 & 0 & 0 & 0 & 0 & 227541 \\
2 & 26821 & 16182 & 0 & 0 & 0 & 0 & 0 & 0 & 207998 \\
3 & 23675 & 30753 & 6798 & 0 & 0 & 0 & 0 & 0 & 189775 \\
4 & 22855 & 36819 & 12138 & 5352 & 0 & 0 & 0 & 0 & 173837 \\
5 & 22005 & 36504 & 11344 & 8163 & 5367 & 0 & 0 & 0 & 167618 \\
6 & 21133 & 34355 & 10301 & 14309 & 5705 & 5102 & 0 & 0 & 160096 \\
7 & 20467 & 32567 & 10548 & 16992 & 7680 & 4663 & 4228 & 0 & 153856 \\
8 & 19405 & 30116 & 10437 & 17560 & 10803 & 6020 & 3947 & 3316 & 149397 \\
\hline
\end{tabular}

TABLE II

Number of PiXels in EACH Class at EACH ITERATION - FP.I

\begin{tabular}{|c|c|c|c|c|c|c|c|c|c|}
\hline Iteration & Class 1 & Class 2 & Class 3 & Class 4 & Class 5 & Class 6 & Class 7 & Class 8 & Rejection class \\
\hline 1 & 134700 & 0 & 0 & 0 & 0 & 0 & 0 & 0 & 116301 \\
2 & 93390 & 58805 & 0 & 0 & 0 & 0 & 0 & 0 & 98806 \\
3 & 74961 & 48825 & 39973 & 0 & 0 & 0 & 0 & 0 & 87242 \\
4 & 63934 & 50222 & 30778 & 29598 & 0 & 0 & 0 & 0 & 76469 \\
5 & 58142 & 44901 & 28036 & 25856 & 22162 & 0 & 0 & 0 & 71904 \\
6 & 52068 & 39529 & 27025 & 28466 & 19353 & 17327 & 0 & 0 & 67233 \\
7 & 44831 & 35445 & 27310 & 28433 & 22625 & 13003 & 16012 & 0 & 63342 \\
8 & 38178 & 32443 & 27764 & 27656 & 23804 & 13461 & 14610 & 13240 & 59845 \\
\hline
\end{tabular}

After one iteration, most of the pixels of the image are rejected and no feature of the image is well discernible. When compared to Fig. 3(a), the pixels of the first class appear to belong to the yellow class in the $H-\alpha$ decomposition (which corresponds to a random surface with medium entropy). This is rather accurate since the pixels belong to fields.

After eight iterations, there are still rejected pixels in the fields areas, especially the top field which is still almost entirely rejected. However, the remaining rejected pixels outline for the most part the urban features of the scene: the two big buildings are clearly identified, as well as the small building next to them, the urban area on the top left, the parking lot on the right side and the dihedrals used for calibration. Even the biggest roads can be seen. Concerning the Gaussian parts, the bottom left field is pretty much identified as only one class but the others are a mixture of several classes and do not really stand out. On forested areas on the right part of the image, most of the area is rejected as well.

2) FP Estimate: For the FP estimate, the classification result after one iteration is encouraging. The number of rejected pixels is reduced compared to the SCM case (see Tables I and II for numbers of pixels in each class at every iteration) and the man-made features already stand out much more. The reduction of rejected pixels means a reduction in false alarms, which proves that the FP estimate is more adapted to the heterogeneous clutter than the SCM. After eight iterations, Fig. 9(b), the results are pretty close to the results of the SCM concerning the buildings and the dihedrals. On the rest of the image, the classification results are very different from the one obtained with the classical methods. Indeed, the patches are much smaller. This is due to the fact that the classification is not made on the power: as the estimation of the covariance is made with the spatial neighbors of the pixel under consideration, on classical methods, there can be stronger reflectors on nearby cases that will bias the classification results on a large area. It is difficult to provide an interpretation at this time since we do not have the ground truth
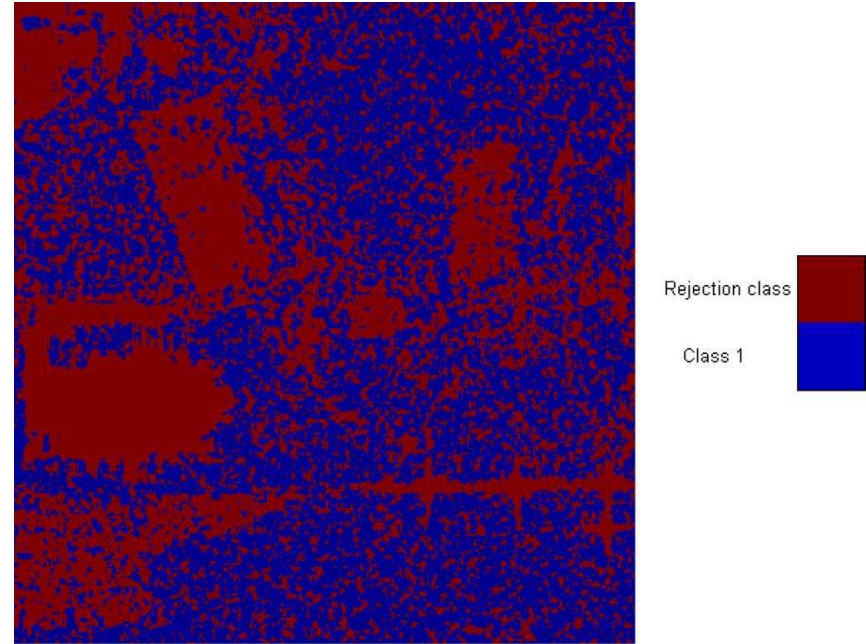

(a)
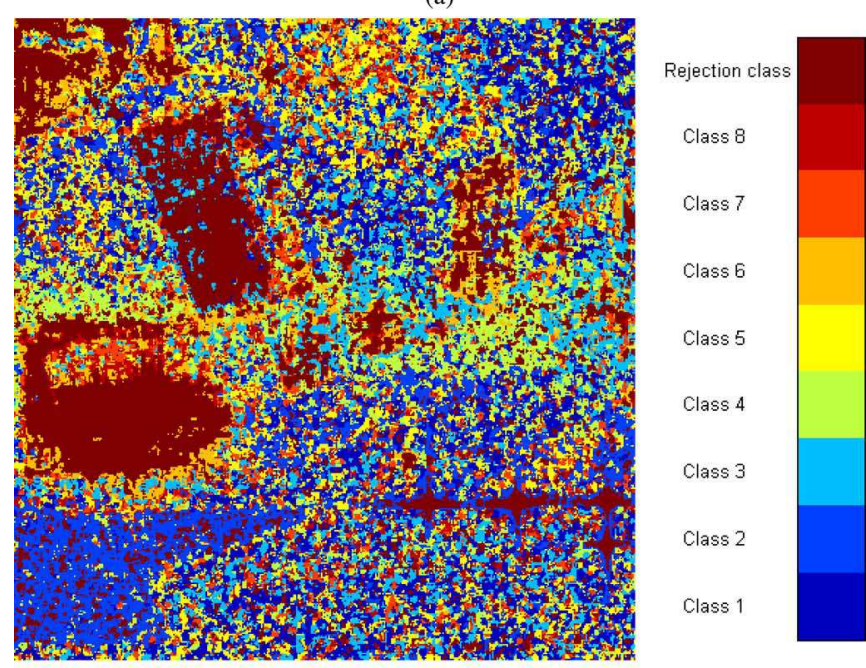

(b)

Fig. 9. Box classifier with FP and associated colormaps. (a) One class + rejection class. (b) Eight classes + rejection class 

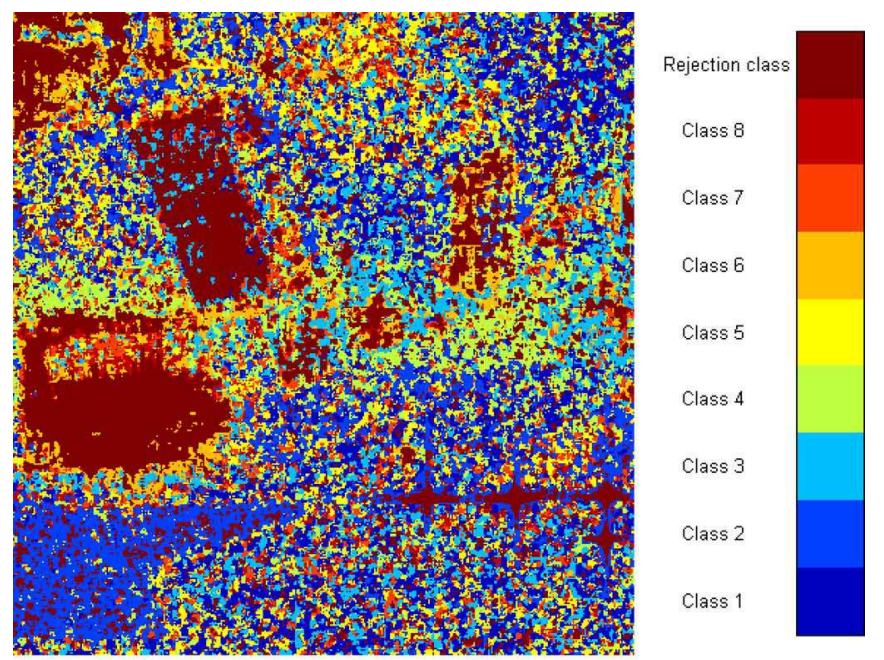

(a)
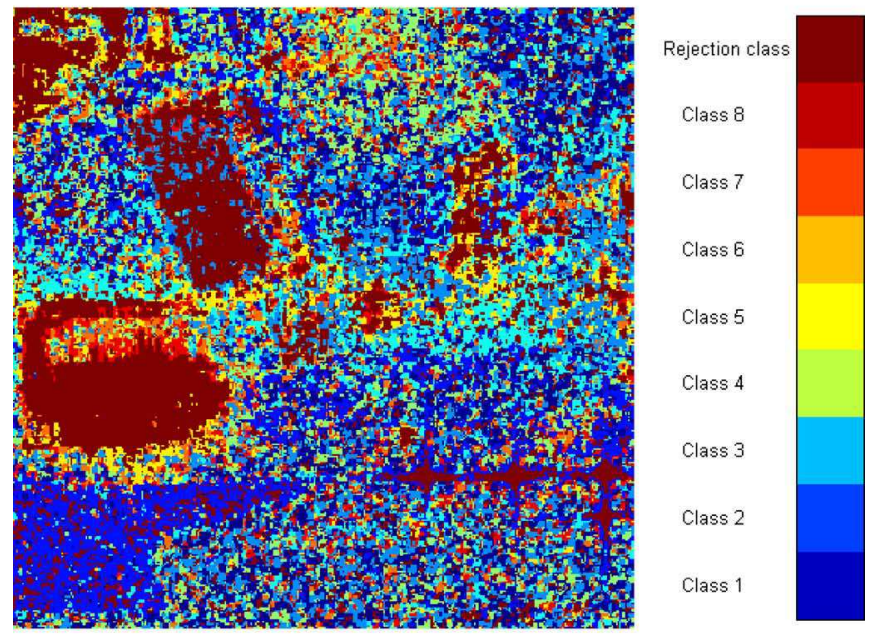

(b)

Fig. 10. Box classifier with FP and associated colormaps. (a) $H-\alpha$ initialization. (b) Random initialization.

data. A comparison with other classification methods is difficult as well, since there are very few classification results on the actual polarimetric information.

Furthermore, as mentioned in Section IV, we selected randomly a large number of pixels ( $\sim 1 / 8$ of the total number) for the initialization to compare this method with the $H-\alpha$ initialization. The result of the algorithm with this random initialization is presented on Fig. 10. As in Section III, using a $H-\alpha$ initialization or a random initialization with a large number of pixels provides the same end results, even if the colors differ. It is thus possible to skip the $H-\alpha$ initialization phase for this scheme as well.

\section{CONCLUSION AND PERSPECTIVES}

This paper presented a rigorous statistical approach for polarimetric SAR data classification. It relies on a simple binary test of equality of covariance matrices which can be applied to both homogeneous and heterogeneous clutter models.

The heterogeneous clutter in POLSAR data was described by the SIRV model. The Fixed Point estimate of the covariance matrix was used to describe the POLSAR data set. It is independent of the texture pdf and is an AML estimator for many stochastic processes obeying the SIRV model. Moreover, it is asymptotically Wishart distributed. Performances of a classical classification procedure designed for Gaussian clutter and of a procedure adapted to the heterogeneous assumption were compared on a high resolution polarimetric data set.

The statistical test was presented and classical distance measures derived as particular cases of the test. The main improvement of this approach is to introduce a critical region which properly rejects pixels that are not close enough to the class centers, contrary to existing methods. The test statistic in the general case has been used as a distance measure in a new algorithm, applied on the POLSAR data set. Results show mainly that heterogeneous pixels are rejected from the rest of the image.

The proposed approach is very general and can be applied to other multidimensional SAR techniques such as polarimetric interferometry or multi-frequency polarimetry. Perspectives include the study of the class centers. They are defined as the mean of all covariance matrices inside a class but, although that may hold for the SCM, it does not for the FP estimate as the fixed point of a sum is not the sum of the fixed points. The geometry of covariance matrices have to be considered more accurately to use the correct center of mass. A short term goal is to compare the results of the classification to the ground truth data in order to have a better analysis.

\section{REFERENCES}

[1] W. M. Boerner, Basic Concepts of Radar Polarimetry and its Applications to Target Discrimination, Classification, Imaging, and Identification Univ. of Illinois, Commun. Lab., Chicago, IL, 1982, EMID-CL-82-05-18-0.

[2] J. Goodman, "Some fundamental properties of speckle," J. Opt. Soc. Amer. A, vol. 66, pp. 1145-1149, 1976.

[3] M. S. Greco and F. Gini, "Statistical analysis of high-resolution SAR ground clutter data," IEEE Trans. Geosci. Remote Sens., vol. 45, no. 3, pp. 566-575, Mar. 2007.

[4] K. J. Sangston and K. Gerlach, "Coherent detection of radar targets in a non-Gaussian background," IEEE Trans. Aerosp. Electron. Syst., vol. 30, no. 2, pp. 330-340, Apr. 1994.

[5] K. Yao, "A representation theorem and its applications to sphericallyinvariant random processes," IEEE Trans. Inf. Theory, vol. IT-19, no. 5, pp. 600-608, Sep. 1973.

[6] S. H. Yueh, J. A. Kong, J. K. Jao, R. T. Shin, and L. M. Novak, "K-distribution and polarimetric terrain radar clutter," J. Electromagn. Waves Applicat., vol. 3, no. 8, pp. 747-768, 1989

[7] V. Anastassopoulos, G. A. Lampropoulos, A. Drosopoulos, and M. Rey, "High resolution radar clutter statistics," IEEE Trans. Aerosp. Electron. Syst., vol. 35, no. 1, pp. 43-60, Jan. 1999.

[8] E. Conte, A. de Maio, and C. Galdi, "Statistical analysis of real clutter at different range resolutions," IEEE Trans. Aerosp. Electron. Syst., vol. 40, no. 3, pp. 903-918, Jul. 2004.

[9] A. Fusco, C. Galdi, G. Ricci, and M. Tesauro, "Fitting a statistical model to SIR-C SAR images of the sea surface," IEEE Trans. Aerosp. Electron. Syst., vol. 40, no. 4, pp. 1179-1190, Oct. 2004.

[10] M. Rangaswamy, "Statistical analysis of the nonhomogeneity detector for non-Gaussian interference backgrounds," IEEE Trans. Signal Process., vol. 53, no. 6, pp. 2101-2111, Jun. 2005.

[11] J. A. Kong, A. A. Swartz, and H. A. Yueh, "Identification of terrain cover using the optimal terrain classifier," J. Electronmagn. Waves Applicat., vol. 2, pp. 171-194, 1988.

[12] H. A. Yueh, A. A. Swartz, J. A. Kong, R. T. Shin, and L. M. Novak, "Optimal classification of terrain cover using normalized polarimetric data," J. Geophys. Res., pp. 15 261-15 267, 1993.

[13] J. J. van Zyl and C. F. Burnette, "Bayesian classification of polarimetric SAR images using adaptive a priori probability," Int. J. Remote Sens., vol. 13 , no. 5 , pp. $835-840,1992$. 
[14] J.-S. Lee, M. R. Grunes, and R. Kwok, "Classification of multi-look polarimetric SAR imagery based on complex Wishart distribution," Int. J. Remote Sens., vol. 15, no. 11, pp. 2299-2311, 1994.

[15] W. L. Cameron, N. Youssef, and L. K. Leung, "Simulated polarimetric signatures of primitive geometrical shapes," IEEE Trans. Geosci. Remote Sens., vol. 34, no. 3, pp. 793-803, May 1996.

[16] E. Krogager, "New decomposition of the radar target scattering matrix," Electron. Lett., vol. 26, no. 18, pp. 1525-1527, Aug. 1990.

[17] A. Freeman and S. Durden, "A three component scattering model to describe polarimetric SAR data," Radar Polarimetry, vol. 1748, pp. 213-225, 1992.

[18] S. R. Cloude and E. Pottier, "An entropy based classification scheme for land applications of polarimetric SAR," IEEE Trans. Geosci. Remote Sens., vol. 35, no. 1, pp. 68-78, Jan. 1997.

[19] J.-S. Lee, M. R. Grunes, T. L. Ainsworth, L.-J. Du, D. L. Schuler, and S. R. Cloude, "Unsupervised classification using polarimetric decomposition and the complex Wishart classifier," IEEE Trans. Geosci. Remote Sens., vol. 37, no. 5, pp. 2249-2258, Sep. 1999.

[20] G. Vasile, J.-P. Ovarlez, F. Pascal, and C. Tison, "Coherency matrix estimation of heterogeneous clutter in high-resolution polarimetric SAR images," IEEE Trans. Geosci. Remote Sens., vol. 48, no. 4, pp. 1809-1826, Apr. 2010.

[21] T. W. Anderson, An Introduction to Multivariate Statistical Analysis. New York: Wiley, 1984.

[22] M. Abramowitz and I. Stegun, Handbook of Mathematical Functions. Gaithersburg: National Bureau of Standards, 1964, vol. AMS 55.

[23] M. Rangaswamy, D. D. Weiner, and A. Ozturk, "Computer generation of correlated non-Gaussian radar clutter," IEEE Trans. Aerosp. Electron. Syst. , vol. 31, no. 1, pp. 106-116, Jan. 1995.

[24] J. B. Billingsley, Ground Clutter Measurements for Surface-Sited Radar Mass. Inst. Technol., Cambridge, 1993, Tech. Rep. 780.

[25] F. Gini and M. Greco, "Covariance matrix estimation for CFAR detection in correlated heavy-tailed clutter," Signal Process., vol. 82, no. 12, pp. 1847-1859, 2002.

[26] E. Conte, A. De Maio, and G. Ricci, "Recursive estimation of the covariance matrix of a compound-Gaussian process and its application to adaptive CFAR detection," IEEE Trans. Signal Process., vol. 50, no. 8, pp. 1908-1915, Aug. 2002.

[27] F. Pascal, Y. Chitour, J.-P. Ovarlez, P. Forster, and P. Larzabal, "Covariance structure maximum-likelihood estimates in compound Gaussian noise: Existence and algorithm analysis," IEEE Trans. Geosci. Remote Sens., vol. 56, no. 1, pp. 34-48, Jan. 2008.

[28] M. S. Bartlett, "Properties of sufficiency and statistical tests," in Proc. R. Soc. London, 1937, vol. 160, pp. 268-282.

[29] G. Box, "A general distribution theory for a class of likelihood criteria," Biometrika, vol. 36, pp. 317-346, 1949.

[30] G. Box, "Problems in the analysis of growth and linear curves," Biometrika, vol. 6, pp. 362-389, 1950.

[31] F. Pascal, J.-P. Ovarlez, P. Forster, and P. Larzabal, "Performance analysis of covariance matrix estimates in impulsive noise," Signal Process., vol. 56, no. 6, pp. 2206-2216, 2008.

[32] K. Conradsen, A. A. Nielsen, J. Schou, and H. Skriver, "A test statistic in the complex Wishart distribution and its application to change detection in polarimetric SAR data," IEEE Trans. Geosci. Remote Sens., vol. 51, no. 1, pp. 4-19, Jan. 2003.

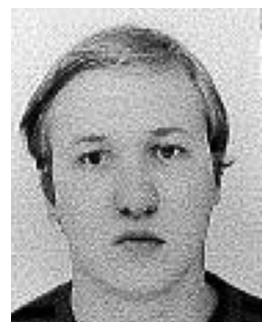

Pierre Formont (S'10) was born in Villeurbanne, France, in 1986. He received jointly the engineering degree from Ecole Nationale Supérieure de l'Eau, l'Energie et l'Environnement (ENSE3), Grenoble, France, and the M.S. degree in "Signal, Images, Speech, Telecom") with merit from Institut National Polytechnique de Grenoble (INPG) and Université Joseph Fourier (UJF), Grenoble, France, in 2009. $\mathrm{He}$ is currently pursuing the Ph.D. degree in image and signal processing at the French Aerospace Lab (ONERA), Palaiseau, France.

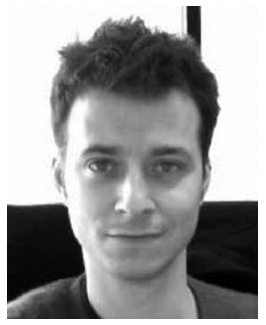

Frédéric Pascal (M’08) was born in Sallanches, France, in 1979. He received the M.S. degree ('Probabilities, Statistics and Applications: Signal, Image et Networks") with merit, in applied statistics from University Paris VII-Jussieu, Paris, France, in 2003 and the Ph.D. degree in signal processing from the University Paris X - Nanterre, advised by Pr. Philippe Forster: "Detection and Estimation in Compound Gaussian Noise" in 2006. This Ph.D thesis was in collaboration with the French Aerospace Lab (ONERA), Palaiseau, France.

From November 2006 to February 2008, he made a postdoctoral position in the Signal Processing and Information Team, Laboratory Système et Applications des Technologies de l'Information et de l'Energie (SATIE), CNRS, Ecole Normale Supérieure, Cachan, France. Since March 2008, he has been an Assistant Professor in the SONDRA Laboratory, SUPELEC, Gif-sur-Yvette, France. His research interests are estimation in statistical signal processing and radar detection.

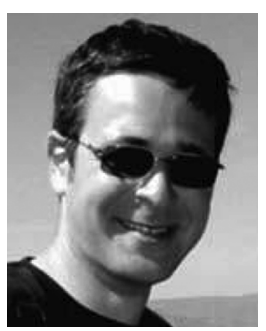

Gabriel Vasile (S'06-M'07) received the M.Eng. degree in electrical engineering and computer science and the M.S. degree in image, shapes, and artificial intelligence from the POLITEHNICA University, Bucharest, Romania, in 2003 and 2004, respectively, and the Ph.D. degree in signal and image processing from Savoie University, Annecy, France, in 2007.

From 2007 to 2008, he was a Postdoctoral Fellow with the French Space Agency (CNES) and was with the French Aerospace Laboratory (ONERA), Palaiseau, France. In 2008, he joined the French National Council for Scientific Research (CNRS), where he is currently a Research Scientist and a member of the Grenoble Image Speech Signal Automatics Laboratory, Grenoble, France. His research interests include signal and image processing, synthetic aperture radar remote sensing, polarimetry, and interferometry.

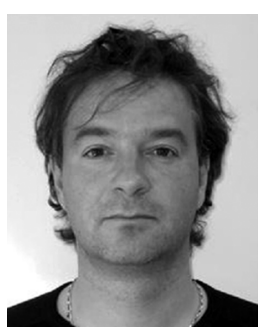

Jean-Philippe Ovarlez (M'06) was born in Denain, France, in 1963. He received jointly the engineering degree from Ecole Supérieure d'Electronique $\mathrm{Au}-$ tomatique et Informatique (ESIEA), Paris, France, and the Diplôme d'Etudes Approfondies degree in Signal Processing from University of Orsay (Paris $\mathrm{XI})$, Orsay, France, and the Ph.D. degree in physics from the University of Paris VI, Paris, France, in 1987 and 1992, respectively.

In 1992, he joined the Electromagnetic and Radar Division of the French Aerospace Lab (ONERA), Palaiseau, France, where he is currently Principal Scientist and member of the Scientific Committee of the ONERA Physics Branch. In January 2008, he joined the French-Singaporean SONDRA Lab on a part-time basis to supervise Signal Processing topics. His current activities of research are centered in the topic of statistical signal processing for radar and SAR applications such as time-frequency, imaging, detection, and parameters estimation.

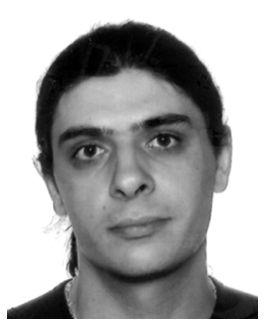

Laurent Ferro-Famil received the Laurea degree in electronics systems and computer engineering, the M.S. degree in electronics, and the Ph.D. degree from the University of Nantes, Nantes, France, in 1996, 1996, and 2000, respectively.

In 2001, he became an Assistant Professor, and, since 2007, he has been an Associate Professor with the University of Rennes 1, Rennes, France, where he is currently a Member of the Radar Polarimetry Remote Sensing Group, Institute of Electronics and Telecommunications of Rennes. His current activities in education are concerned with analog electronics, digital communications, microwave theory, and polarimetric SAR remote sensing. He is particularly interested in polarimetric SAR signal processing, radar polarimetry theory, and natural media remote sensing using multibaseline PolInSAR data, with application to classification, electromagnetic scattering modeling and physical parameter retrieval, time-frequency analysis, and 3-D reconstruction of environments. 